\title{
Balkanmediävistik als Forschungskonzept ${ }^{1}$
}

\author{
Oliver Jens Schmitt \\ Wien
}

Die mittelalterliche Geschichte des Balkans ist ganz überwiegend ein Gegenstand regionaler Nationalhistoriographien, ${ }^{1}$ d.h. der Spezialforschung im Balkan selbst. Außerhalb des Balkans ist das Interesse am Thema weitgehend erloschen. Doch ist das Interesse an Nationalgeschichte im Mittelalter gleichzusetzen mit einer Balkanmediävistik? Dieser Vortrag möchte eine Reihe von Fragen an einen Gegenstand stellen, den es zunächst genauer zu umreißen gilt. Mediävistik selbst bedarf im Kontext der Balkangeschichte nur scheinbar keiner terminologischen Klärung. Gemeinhin lässt man das Mittelalter im Balkan mit der Einwanderung slawischer Gruppen in das römische Reich beginnen und mit der osmanischen Eroberung des Raumes enden. Doch was für den inneren Balkan ein Endzeitalter ist - d.h. das 14.-15. Jahrhundert - bedeutet für die orthodoxen Fürstentümer Walachei und Moldau Anfang und Aufstieg - entsprechend setzt eine an Herrschaftsbildung orientierte rumänische Historiographie erst im 14. Jahrhundert ein und führt ihr Mittelalter, ihre ,istorie medie", bis zum Beginn der Phanariotenzeit hinauf. Was die räumliche Dimension des Begriffs Balkanmediävistik betrifft, also den Balkan, wird diskutiert, seit der Terminus in die Welt gesetzt wurde. Er wird hier wertneutral verwendet, eine Bemerkung, die Eulen nach Athen trägt an einem Institut für Balkanologie. Doch was Balkan in einem mittelalterlichen Kontext bedeutet und weswegen der Begriff als wissenschaftliche Kategorie sinnvoll sein kann, wurde bislang kaum

\footnotetext{
${ }^{1}$ Angesichts des essaiartigen Charakters des Texts und der Breite der Thematik wird auf eine detaillierte Bibliographie verzichtet.
}

diskutiert. Umfangreiche Balkangeschichten im Mittelalter gibt es nur wenige. Die englischsprachige Synthese von John V.A. Fine bietet eine Addition von (proto-)nationalen Räumen zwischen Kroatien und Griechenland, Albanien und der bulgarischen Donaugrenze, wobei das Schwergewicht der Darstellung entsprechend der Forschungskompetenz des Verfassers, im Westen der Balkanhalbinsel liegt. Eine eigentliche Definition seines Untersuchungsraumes bietet Fine nicht ${ }^{2}$. Florin Curta, Verfasser eine forschungsbasierten Synthese bis 1250, meidet den Balkanbegriff; sein Südosteuropa reicht von Dalmatien bis in das heutige Rumänien, wobei im Hintergrund die rumänische Südosteuropakonzeption der Zwischenkriegszeit durchschimmert ${ }^{3}$. Hristo Matanov veröffentlichte eine bulgarische Synthese, in der erdie in einem balkanmediävistischen Zusammenhang wohl jüngste Raumdefinition bietet $^{4}$ : Matanovs Balkan reicht von Dalmatien bis in die Walachei, umfasst also die orthodoxe byzantinisch geprägte Welt einschließlich ihrer äußersten westlichen Peripherie, dem kaum byzantinisch durchdrungenen Bosnien. Dalmatien ist voller Bestandteil dieses Balkankonzepts, das aber die internationale Raumdiskussion aufgreift und mit breiten Kontaktzonen arbeitet. Überhaupt konzipiert Matanov den Balkan weniger als „Kreuzung“ oder „Brücke“ zwischen Kulturen, sondern als „Kontaktzone“ von Gesellschaften, Sprachen und Religionen ${ }^{5}$. Im folgenden soll von einem dem Matanov'schen Modell entsprechen-

\footnotetext{
${ }^{2}$ Fine 1983; ders. 1987.

${ }^{3}$ Curta 2006

${ }^{4}$ Matanov 2002.

${ }^{5}$ Matanov 2002, 7
} 
den Raum, also einem „byzantinischen Commonwealth" (Dimitri Obolensky) ausgegangen werden. Gibt es nun in diesem Rahmen eine Balkanmediävistik? Die Antwort muss im Kontext innerregionaler Versuche, Balkangeschichte $\mathrm{zu}$ schreiben, erfolgen. Diese innerregionalen Balkanstudien folgten auch politischen Strömungen und Konjunkturen, vom Balkanföderalismus, dem Balkanband bis zu Mächtekonstellationen im Kalten Krieg, schließlich den getrennten Wegen nach Brüssel in der Gegenwart. Diese Form der Balkangeschichtsschreibung konstruierte einen geschlossenen Balkanraum in Abgrenzung $\mathrm{zu}$ als bedrohlich empfundenen Grossmächten; und wo diese Art der Geschichtsbetrachtung betrieben wurde, allen voran in Bukarest und Belgrad, schwangen nationale Hegemoniepläne innerhalb dieses Balkans der Balkanvölker mit. Dem politischen Rahmen zum Trotz unterschied eines innerregionale Zusammenarbeit von Historikern deutlich vom ausserregionalen Blick: denn dieser konzentrierte sich nach 1945, besonders aber nach 1989/91 zunehmend ausschliesslich auf das 20. Jahrhundert. Innerregionale Forschungsinitiativen hingegen gaben dem Mittelalter und der osmanischen Periode ein starkes Eigengewicht, und dies lag nicht nur an der irenischen balkanistischen Tendenz dieser Bemühungen, sondern auch an der Bedeutung, die diesen Epochen zugemessen wird: so darf man von einer unterschiedlichen Tiefe und einer besonderen Funktion der Zeitachse sprechen, wenn man inner- und ausserregionale historische Forschung vergleicht. Fragt man nun nach Ergebnissen der innerregionalen Zusammenarbeit, ist die Ernte freilich bescheiden: wenige offizielle UNESCO-Bände, die oftmals additiv nationale Standpunkte wiedergeben. Das weitgehende Scheitern balkanmediävistischer Bemühungen ist ablesbar

1) An dem fast völligen Fehlen des Balkans in der europäischen Mediävistik, die an den Grenzen des alten römisch-deutschen Reiches, im besten Falle, den Grenzen des Königreichs Ungarn endet.

2) An der Dominanz der nationalen Perspektive der meisten Forschungen im Balkan selbst. Einen raumübergreifenden Blick nehmen nur die imperialen Wissenschaften, d.h. Byzantinistik und Osmanistik ein, doch folgen sie einerseits stark dem Blick ihrer Quellen - konstruieren den
Balkan also als Peripherie - oder bleiben oftmals nationalen Perspektiven verhaftet, zumindest bei der Bestimmung der Untersuchungsräume.

Mit anderen Worten: Balkanmediävistik existiert(e) als Forschungsprogramm, wenn auch bisweilen nur vage umschrieben, doch weder inner- noch ausserregional gewann sie je an nachhaltigem Schwung. Dann ist das Konzept eben nicht tragfähig genug oder der wissenschaftliche Erkenntnisgewinn zu bescheiden, mag man einwenden.

Warum braucht es überhaupt eine Balkanmediävistik? Diese Frage einer Veröffentlichung eines Instituts für Balkanstudien zu stellen, mag beinahe ketzerisch erscheinen - um eine Diskussion anzustossen, ist sie aber nötig.

1) Forschungspragmatische Gründe: den Nationalhistoriographien des Balkans gelingt es nur in Ansätzen, in europäischen Diskussionen Gehör zu finden. Die Ignoranz der „Westmediävistik" gegenüber dem Balkan ist wohl noch grösser als jene der allgemeinen Neuzeithistoriker. Nur gemeinsame grossräumige Forschungsinitiativen und entsprechende vorgestellte Ergebnisse können hier zumindest in einem ersten Schritt Abhilfe schaffen - dafür müssen die engen nationalen Zunftgrenzen überschritten werden.

2) Es geht darum, grosse Fragen der allgemeinen Mediävistik aufzugreifen. Einzelne Forscher haben dies schon längst getan, doch ein breiterer Austausch, wie er z.B. durch die Rezeption der Annales-Schule im Jugoslawien der 60er/70er Jahre erfolgt ist, fehlt leider weitgehend. Die politische Fragmentierung der letzten 20 Jahre ist nicht selten auch von Reprovinzialisierung und Selbstisolierung geprägt, was besonders für Institutionen, weniger für einzelne Forscherindividuen gilt.

3) Wohl gemerkt: es wird hier nicht ein mechanischer Methodentransfer von Westen nach Ost verlangt. Vielmehr sollte eine europäische Verschränkung das Ziel sein, wobei die Westmediävistik viel von den fachlich hochstehenden Arbeiten der Mediävistiken im Balkanraum lernen kann. Die damit einhergehende Europäisierung ist 1) kein politisches, sondern ein wissenschaftlich-intellektuelles Vorhaben und 2) hat sich die Westmediävistik durch eine Öffnung nach Südosten ebenfalls zu europäisieren. Nur so können raumübergreifende grosse Fra- 
gestellungen der mittelalterlichen europäischen Geschichte bewältigt werden.

4) Dies bedeutet nicht, dass die Balkanmediävistiken von Strömungen der Westmediävistik völlig abgekoppelt sind. Gerade bei der Erforschung der Meta-Ebene, d.h. der neuzeitlichen politischen Instrumentalisierung mittelalterlicher Geschichte, z.B. in Schulbüchern, wurde im Balkan in den letzten jahren viel geleistet. Doch die Aufarbeitung politisch schwieriger Mittelalterbilder ersetzt genuine Forschung zum Mittelalter selbst nicht.

5) Wo nun läge das Potential einer Balkanmediävistik? Es liegt meines Erachtens vorwiegend in zwei Bereichen:

- Einer innerregionalen Verschränkung der im nationalen Rahmen erzielten Ergebnisse.

- Einer Verschränkung auf gesamteuropäischer Ebene, aber auch mit anderen Bezugsräumen wie Anatolien, dem Ostmittelmeer- und dem Schwarzmeerraum.

Beides ist gewiss nicht spezifisch für die mittelalterliche Geschichte, sondern trifft für die Geschichte des Balkans auch allgemein zu. Die innerregionale Verschränkung speist sich primär aus dem Vergleich, aber eben nicht nur - auch die Balkanmediävistik bewegt sich im Spannungsfeld gesamtbalkanischer Gesellschaftsund Kulturphänomene und regionaler Entwicklungen. So ist weder eine blosse Addition nationaler Fallbeispiele zielführend noch eine von Konflikten bereinigte und gleichsam geglättete und homogenisierte Erzählung. Innerregionaler Vergleich und Verschränkung darf auch nicht bedeuten, den Balkanraum in der Betrachtung $\mathrm{zu}$ isolieren und ihn aus anderen Raumbezügen herauszulösen. Welche Fragen könnte die Balkanmediävistik nun gewinnbringend angehen? Ein gesamtbalkanisches Phänomen sind jene Veränderungen, die mit dem Rückgang oder dem Aufstieg grosser Reiche in Verbindung stehen. Die grundlegenden ethnischen und soziokulturellen Veränderungen des frühen Mittelalters sind nicht hinreichend geklärt und können nur durch einen grossregionalen Bezug und die Zusammenarbeit mit Linguistik und Archäologie untersucht werden. In allen Ländern der Region schreitet die Einzelforschung zwar voran, doch ist die Fragmentierung der Evidenz ein eigentliches Hindernis für grössere Neuerkenntnisse. Die Balkanmediävistik hätte hier in eine der lebhaftesten Debatten der europäischen Mediävistik, die Frage nach der Entstehung des mittelalterlichen Europas, die Deutung des Niedergangs des Römischen Reiches, eine gewichtige Stimme einzubringen. Seit Mommsen fehlt eine antike Geschichte des Balkans, und die Verklammerung von Antike und Mittelalter wird nur von wenigen Historikern bewältigt ${ }^{6}$. Hier hätte eine Balkanmediävistik auch der Transitionsforschung viel zu bieten. Dies gilt auch für einen zweiten Themenbereich, die osmanische Eroberung des Balkans, die in Forschung und Deutung ebenso zersplittert ist, disziplinär wie national. Der diachrone Vergleich mit dem späten Rom bietet sich hier ebenso an wie der Vergleich mit der spanischen Reconquista oder der Ausdehnung des Moskauer Grossfürstentums in muslimische Chanate von der Wolga bis nach Sibirien. Der Balkan als Kontaktraum etwa zwischen den Kirchen von Rom und Konstantinopel ist in den nationalen Historiographien gut präsent, die Stellung des gesamten Balkans im europäischen Rahmen tritt aber nur selten hervor. Doch ist es just die Religionsgeschichte, in welcher der Balkan am besten an die Westmediävistik angekoppelt ist - ich meine die Bogomilen und die Bosnische Kirche, die von westlichen Häresieforschern stets mit Interesse betrachtet wurden. Eine echte Verschränkung südosteuropäischer und westeuropäischer Perspektiven gelang aber nur sehr wenigen Forschern, am ehesten wohl dem tschechischen Historiker Milan Loos? ${ }^{7}$. Fast ganz isoliert ist demgegenüber die Sozial- und Wirtschaftsgeschichte, die in viele einzelne, untereinander kaum verbundene Forschungszweige zerfällt, einmal die Nationalhistoriographien, dann die Forschungen zu den italienischen Handelsrepubliken Genua und Venedig, zu Dubrovnik. Bis heute ist die Frage nach der Stellung des Balkanraums in der mittelalterlichen europäischen Wirtschaft nicht in vertiefter Form behandelt. Und damit ist auch eine der wichtigsten Fragen berührt, die im nationalen Rahmen sehr wohl thematisiert werden:

\footnotetext{
${ }^{6}$ Angestrebt wird eine Wiedereingliederung der römischen Kaiserzeit im „Handbuch der Geschichte Südosteuropas“, das vom Regensburger Institut für Ost- und Südosteuropastudien IOS betreut wird. Gemeinsam mit dem Althistoriker Fritz Mitthof (Wien) zeichnet der Verfasser dieser Zeilen für den ersten Band verantwortlich. ${ }^{7}$ Loos 1974.
} 
die Frage nach Peripherisierung oder Teilhabe des Balkanraums an europäischen Entwicklungen des Mittelalters. Dabei geht es nicht um ein Modell, das mit einer idealen Norm, entwickelt am Beispiel Nordwesteuropas, und abgestuften Peripherien operiert - sondern um die Frage, ob es neben dualistischen (oder vermeintlich dualistischen) Strömungen auch andere Zusammenhänge gab, die den Balkan eng an andere Teile Europas anschlossen. Migration wäre ein solches Thema $^{8}$ - wiederum im Detail national gut erforscht - im europäischen Geschichtsbild aber fehlend; höfische Kultur und die Zirkulation von Kulturgüter wie Handschriften; bedeutsam wäre ein breiterer Blick auf Kommunikation und Verkehr, aber auch die politische Geschichte könnte sowohl innerbalkanische Zusammenhänge - z.B. die gegen die Osmanen gerichteten Allianzen des 14. und frühen 15. Jahrhunderts - genauer in den Blick nehmen, ebenso die Verwobenheit der Balkanherrschaften mit dem mittel- und südeuropäischen Staatensystem - wiederum national gut erforscht, im übergreifenden Vergleich aber kaum vorhanden. Balkanmediävistik soll die nationalen Zugänge der Mittelalterforschung nicht ersetzen, sondern vielmehr diesen neue Forschungsperspektiven eröffnen. Sie stützt sich dabei auf sehr gut ausgebildete nationale Schulen -es braucht also keinen wissenschaftlichen Umsturz, sondern eine Diskussion, die das Bewusstsein um die Chancen einer räumlichen und methodischen Öffnung schärft. Dass dies im Balkan selbst erfolgen muss, liegt auf der Hand, denn ausserregional ist die Mittelalterforschung zum Balkan fast ganz zum Erliegen gekommen. Ein balkanmediävistischer Blick, der die Region in ihrer Vielfalt auf der mentalen Landkarte der europäischen Mediävistik verankert, würde aber dieses Interesse wieder wecken. Und so sollte es heissen: der Balkan nicht den Mediävisten im Balkan, sondern der Balkan den Balkanmediävisten.

\footnotetext{
${ }^{8}$ Ein grundlegendes Forschungsprogramm bei Sundhaussen 2007, 422-477; eine Skizze zur frühen Neuzeit bietet Katsiardi - Hering 2001, 125-148.
}

\section{Sažetak}

\section{Balkanska medijevistika kao istraživački koncept}

Srednjovjekovna istorija Balkana pisana je prvenstveno u okvirima nacionalnih historiografija određenih država, i to uglavnom u formi nacionalne istorije. Medijevistička kompetencija izvan regiona u zadnjih trideset godina je u znatnoj mjeri opala. Isto tako primijetan je nizak nivo komunikacije ne samo u regionalnoj medijevistici, već i u kontekstu istorije srednjeg vijeka u Evropi uopšte. Imajući to u vidu, razmatra se u kojoj mjeri bi jače povezivanje regionalnog istraživanja te pojačano koncepciono određenje predmeta istraživanja moglo doprinijeti boljem uvidu u regionalna istraživanja. U referatu se takođe diskutuje o odnosu balkanske medievistike prema "imperijalnim" disciplinama - bizantologiji i osmanistici.

\section{Literaturverzeichnis}

Curta, F. 2006, Southeastern Europe in the Middle Ages, 500-1250, Cambridge 2006.

Fine, J. V. A. 1983, The Early Medieval Balkans, Ann Arbor 1983.

Fine, J. V. A. 1987, The Late Medieval Balkans, Ann Arbor 1987.

Hering, O. 2001, Migrationen von Bevölkerungsgruppen in Südosteuropa vom 15. bis zum Beginn des 18. Jahrhunderts, Südost-Forschungen, 59/60, 2000/2001, 125-148.

Loos, M. 1974, Dualist Heresy in the Middle Ages, Prag 1974.

Matanov, H. 2002, Srednovekovnite Balkani: istoričeski očerci, Sofia 2002.

Sundhaussen, H. 2007, Geschichte Südosteuropas als Migrationsgeschichte: Eine Skizze, Südost-Forschungen 65/66, 2006/2007, 422-477. 